\title{
A current driven electromagnetic mode in sheared and toroidal configurations
}

\author{
István Pusztai $^{1,2}$, Peter J Catto ${ }^{1}$, Felix I Parra ${ }^{1,3}$, \\ Michael Barnes ${ }^{1,4}$ \\ ${ }^{1}$ Plasma Science and Fusion Center, Massachusetts Institute of Technology, \\ Cambridge, MA 02139, USA \\ 2 Department of Applied Physics, Chalmers University of Technology and \\ Euratom-VR Association, SE-41296 Göteborg, Sweden \\ ${ }^{3}$ Department of Physics, University of Oxford, Oxford, OX1 3PU, UK \\ ${ }^{4}$ Department of Physics, University of Texas at Austin, Austin, TX 78712, USA \\ E-mail: pusztai@chalmers.se
}

\begin{abstract}
The induced electric field in a tokamak drives a parallel electron current flow. In an inhomogeneous, finite beta plasma, when this electron flow is comparable to the ion thermal speed, the Alfvén mode wave solutions of the electromagnetic gyrokinetic equation can become nearly purely growing kink modes. Using the new "low-flow" version of the gyrokinetic code GS2 developed for momentum transport studies [Barnes et al 2013 Phys. Rev. Lett. 111 055005], we are able to model the effect of the induced parallel electric field on the electron distribution to study the destabilizing influence of current on stability. We identify high mode number kink modes in GS2 simulations and make comparisons to analytical theory in sheared magnetic geometry. We demonstrate reassuring agreement with analytical results both in terms of parametric dependences of mode frequencies and growth rates, and regarding the radial mode structure.
\end{abstract}




\section{Introduction}

The radial gradient of electric current represents a source of free energy in fusion plasmas which can drive or modify instabilities. For a sufficiently strong current gradient, kink modes can be destabilized. The criterion for destabilization in a screw pinch was derived in [1] with a magnetohydrodynamic formulation (there referred to as the screw-instability) for high mode numbers.

Low mode number kink modes are important for the internal stability of tokamaks. The $n=m=1$ internal kink mode is believed to be responsible for the sawtooth instability [2]. Most of the work done on these modes uses a fluid formalism. However, accounting for kinetic effects is important to reproduce all details of the evolution of such instabilities. For instance, finite electron inertia can assist collisionless reconnection that can modify the dynamics of $m=1$ internal kinks, as well as their coupling to ion sound waves, as discussed in [3]. In addition, considering fluid ions and kinetic electrons, collisional and diamagnetic effects on the $m=1$ mode were studied in [4]. In spite of the recognized importance of kinetic effects on kink modes, at present there are only a limited number of numerical studies in the literature which employ gyrokinetic [5, 6] simulations. The first is a simulation of a sawtooth crash that was reported in [7], where a particle-in-cell (PIC) code neglecting ion finite Larmor radius (FLR) effects was used to model the instability for straight field lines. More recently, ideal-MHD internal kink and collisionless $m=1$ tearing mode simulations were performed in a screw pinch geometry in [8] with the PIC code GYGLES 9]. Gyrokinetic studies on modifications to kinetic instabilities due to parallel current are also very limited. In [10] the effects of equilibrium current on reversed shear Alfvén eigenmodes is studied using the PIC code GTC [11]. Moreover, the continuum gyrokinetic code GENE [12] is used in [13] to study magnetic reconnection, where alternating current sheets are modeled in a periodic slab configuration. Linear gyrokinetic simulations of tearing modes in the collisional-collisionless transitional regime in a slab geometry are presented in [14] using the AstroGK code [15]. These last two references introduce the parallel current as a

first-order gyrokinetic perturbation, rather than an unperturbed drive term (part of the background distribution) as we do in this article.

It is of interest to further develop our kinetic simulation capability for current driven instabilities. Using the tools available in the new version of the gyrokinetic code GS2 [16], developed for intrinsic rotation studies in tokamaks [17], we are now able to model the destabilizing effect of the modifications to the non-fluctuating electron distribution function due to an induced electric field in a tokamak. In particular, current driven modes can be studied using this continuum gyrokinetic code, as demonstrated herein through simulations of high mode number kink modes with GS2. The Gs2 simulations presented here are radially local (flux tube), which inherently assumes a separation of the parallel and perpendicular scale lengths of perturbed quantities. Accordingly, in GS2, only high mode number modes can be simulated, while global modes, such as the $n=m=1$ mode are beyond the region of applicability of local codes. The simulations 
are done in toroidal geometry and no simplifying assumptions (i.e., regarding finite Larmor radius effects, kinetic treatment of different species, particle drifts etc.) are made to the Maxwell-gyrokinetic system apart from those consistent with the lowest order local gyrokinetic treatment. The new feature is the treatment of the modification to the electron distribution due to the induced electric field as an unperturbed drive term entering as a part of the non-fluctuating distribution. The code results will be shown to be in very good agreement with the analytical calculations we present.

The subsequent sections are organized as follows. First, in Sec. 2 the electromagnetic gyrokinetic equations are derived in toroidal geometry in the presence of an induced parallel electron current. In Sec. 3, the dispersion relation of the high mode number kink modes is derived in shearless toroidal geometry. The effects of magnetic shear and the eigenmode structure are discussed in Sec. 4. Finally, in Sec. 5 the analytical results are compared to GS2 simulations, before we conclude in Sec. 6.

\section{Electromagnetic gyrokinetic equations with induced current}

The induced electric field driven part of the non-fluctuating electron distribution is similar to the solution of the Spitzer problem, $C_{l}\left[f_{\text {Spitz }}\right]=-\left(e_{e} / T_{e}\right) E_{I} v_{\|} f_{M e}$, where $C_{l}$ is the linearized electron collision operator, $f_{M a}=n_{a}\left[m_{a} /\left(2 \pi T_{a}\right)\right]^{3 / 2} \exp \left[-m_{a} v^{2} /\left(2 T_{a}\right)\right]$ is the Maxwell distribution, with the density $n_{a}$, temperature $T_{a}$, mass $m_{a}$ and charge $e_{a}$ of species $a$ (ions and electrons are denoted with the indices $a=i$ and $e$, respectively). Furthermore, $E_{I}$ denotes the induced parallel electric field, $v^{2}=\mathbf{v} \cdot \mathbf{v}$ and $v_{\|}=\mathbf{v} \cdot \mathbf{b}$, with $\mathbf{v}$ the velocity and $\mathbf{b}$ the unit vector in the direction of the equilibrium magnetic field $\mathbf{B}_{0}$. The Spitzer function is proportional to $v_{\|}$, but it may have a non-trivial speed dependence. However, as it will be shown later through simulations, the exact velocity space structure of $f_{\text {Spitz }}$ is unimportant for the instability to be investigated here. Therefore, the induced electric field effects will be modeled simply by allowing for a parallel drift velocity.

To derive the linearized gyrokinetic equation it is convenient to use the unperturbed total energy, $E=v^{2} / 2+\left(e_{a} / m_{a}\right) \phi_{0}$, the magnetic moment, $\mu=v_{\perp}^{2} /\left(2 B_{0}\right)$, and the canonical angular momentum $\psi_{*}=\psi-\left(m_{a} c / e_{a}\right) R \hat{\zeta} \cdot \mathbf{v}$ as phase-space variables. Here, $v_{\perp}^{2}=v^{2}-v_{\|}^{2}, B_{0}=\left|\mathbf{B}_{0}\right|, \phi_{0}$ is the non-fluctuating part of the electrostatic potential, $c$ denotes the speed of light, $R$ is the major radius, $2 \pi \psi$ is the poloidal magnetic flux, and $\hat{\zeta}=\nabla \zeta /|\nabla \zeta|$, with the toroidal angle $\zeta$ and $R|\nabla \zeta|=1$. The unperturbed Vlasov operator $d_{t} \doteq \partial_{t}+\mathbf{v} \cdot \nabla+\left[\left(e_{a} / m_{a}\right) \mathbf{E}_{0}+\Omega_{a} \mathbf{v} \times \mathbf{b}\right] \cdot \nabla_{v}$ acting on functions of only $E$ and $\psi_{*}$ vanishes in a toroidally symmetric system which we shall consider. We have introduced $\Omega_{a}=e_{a} B_{0} /\left(m_{a} c\right)$, with $\mathbf{E}_{0}=-\nabla \phi_{0}+E_{I}$. The time independent piece of the distribution functions should be close to

$$
f_{* a}\left(\psi_{*}, E\right)=\eta_{* a}\left(\frac{m_{a}}{2 \pi T_{* a}}\right)^{3 / 2} \exp \left[-\frac{m_{a} E}{T_{* a}}\right],
$$

where $T_{* a}=T_{a}\left(\psi \rightarrow \psi_{*}\right)$, and the pseudo-density is $\eta_{* a}=n_{* a} \exp \left[e_{a} \phi_{0 *} / T_{* a}\right]$ with $\phi_{0 *}=\phi_{0}\left(\psi \rightarrow \psi_{*}\right)$ and $n_{* a}=n_{a}\left(\psi \rightarrow \psi_{*}\right)$. Note that $\phi_{0}, n_{a}$ and $T_{a}$ are assumed to be 
flux functions. We consider $\phi_{0}=0$. By construction, $f_{* a}$ reduces to a Maxwellian as $\psi_{*} \rightarrow \psi$.

In order to account for the electron flow due to the induced electric field, we model the non-fluctuating electron distribution by

$$
f_{0 e}=f_{* e}\left(\psi_{*}, E\right)+f_{s}\left(\mathbf{R}_{e}, E, \mu\right)
$$

where $f_{s}=-m_{e} v_{\|} u f_{M e} / T_{e}, \mathbf{R}_{a}=\mathbf{r}+\Omega_{a}^{-1} \mathbf{v}_{\perp} \times \mathbf{b}$ is the particle guiding center, and $\mathbf{r}$ is the particle position. Furthermore, $\mathbf{v}_{\perp}=\mathbf{v}-v_{\|} \mathbf{b}$, and the parallel electron flow velocity is $-u$, where $u>0$ is allowed to be comparable to the ion thermal speed $v_{i}=\left(2 T_{i} / m_{i}\right)^{1 / 2}$, and the sign of $u$ is chosen so that the unperturbed current density is $j_{0}=e n_{e} u$. For the electron flow to be divergence free, $u \propto B_{0}$.

The linearized kinetic equation for the fluctuating part of the electron distribution $f_{1 e}$ can be written as

$$
d_{t} f_{1 e}=-\frac{e_{e}}{m_{e}}\left(\mathbf{E}_{1}+\frac{1}{c} \mathbf{v} \times \mathbf{B}_{1}\right) \cdot \nabla_{v} f_{0 e}
$$

where collisions are neglected since we are interested in the tokamak core, where the collision frequency is small. The fluctuating parts of the electric and magnetic fields are denoted by $\mathbf{E}_{1}$ and $\mathbf{B}_{1}$,respectively. We note, that the induced electric field $E_{I}$ is accounted for by retaining its effect on the non-fluctuating distribution, i.e. keeping $f_{s}$ in $f_{0 e}$. The induced electric field is negligible in the $d_{t}$ term of (3), since electron-ion drag requires it to be the same order as a collisional correction.

We represent the perturbed vector potential as $\mathbf{A}_{1}=A_{\psi} \nabla \psi+A_{\theta} \nabla \theta+A_{\zeta} \nabla \zeta$, and work in the Coulomb gauge $\left(\nabla \cdot \mathbf{A}_{1}=0\right)$. Using $\mathbf{E}_{1}=-\nabla \phi_{1}-c^{-1} \partial_{t} \mathbf{A}_{1}$ we obtain

$$
\begin{aligned}
& \left(\mathbf{E}_{1}+\frac{1}{c} \mathbf{v} \times \mathbf{B}_{1}\right) \cdot \nabla_{v} f_{* e}= \\
& -\left[\mathbf{v} \cdot \nabla \phi_{1}+\frac{\mathbf{v}}{c} \cdot\left(\nabla \psi \frac{\partial A_{\psi}}{\partial t}+\nabla \theta \frac{\partial A_{\theta}}{\partial t}+\nabla \zeta \frac{\partial A_{\zeta}}{\partial t}\right)\right] \frac{\partial f_{* e}}{\partial E} \\
& +\frac{m_{e}}{e_{e}}\left[c \frac{\partial \phi_{1}}{\partial \zeta}+\frac{d A_{\zeta}}{d t}-\mathbf{v} \cdot\left(\nabla \psi \frac{\partial A_{\psi}}{\partial \zeta}+\nabla \theta \frac{\partial A_{\theta}}{\partial \zeta}+\nabla \zeta \frac{\partial A_{\zeta}}{\partial \zeta}\right)\right] \frac{\partial f_{* e}}{\partial \psi_{*}},
\end{aligned}
$$

where we define $\partial_{t} A_{\zeta}+\mathbf{v} \cdot \nabla A_{\zeta}=d_{t} A_{\zeta}$. At this point we may neglect finite orbit width corrections to the kinetic equation by replacing $\partial_{E} f_{* e}$ by $\partial_{E} f_{M e}$ and $\partial_{\psi *} f_{* e}$ by $\partial_{\psi} f_{M e}$.

The preceding analysis for $f_{* e}$ is essentially exact, however, we simplify the analytic treatment for $f_{s}$ by considering large aspect ratio $\epsilon=r / R \ll 1$ tokamak magnetic geometry with low normalized pressure $\beta_{i}=8 \pi p_{i} / B_{0}^{2} \ll 1$, where $r$ is the minor radius and $p_{i}=n_{i} T_{i}$ is the ion pressure. We assume that the gyrokinetic ordering is satisfied by any perturbed quantity $Q$, namely $\mathbf{b} \cdot \nabla Q \ll|\nabla Q|, 1 / L \ll|\nabla \ln Q|$, and $f_{1 a} / f_{0 a} \sim e_{a} \phi_{1} / T_{a} \ll 1$, where $L$ represents the perpendicular scale length of background plasma parameters. Additionally, we assume the $-\mathbf{b} \cdot \nabla \phi_{1}$ and the $-\partial_{t} A_{\|} / c=-\mathbf{b} \cdot \partial_{t} \mathbf{A}_{1} / c$ parts of $E_{\|}=\mathbf{b} \cdot \mathbf{E}_{1}$ to be comparable in magnitude.

Next we consider $\nabla_{v}$ acting on $f_{s}$. Neglecting the $\nabla_{R} v_{\|}$term and the poloidal variation of $u$ as small in $\epsilon$, gives

$$
\nabla_{v} f_{s}=-\frac{v_{\|}}{\Omega_{e} B_{0}}\left(I \mathbf{B}_{0}-R^{2} B_{0}^{2} \nabla \zeta\right) \frac{\partial}{\partial \psi}\left(\frac{m_{e} u f_{M e}}{T_{e}}\right)-\frac{m_{e} u f_{M e}}{T_{e}} \mathbf{b}-\mathbf{v} \frac{m_{e} v_{\|} u}{T_{e}} \frac{\partial f_{M e}}{\partial E},
$$


where $\mathbf{B}_{0}=I \nabla \zeta+\nabla \zeta \times \nabla \psi$ and we use $\mathbf{B}_{0} \times \nabla \psi=I \mathbf{B}_{0}-R^{2} B_{0}^{2} \nabla \zeta$. We introduce the thermodynamic forces $F_{1 a}=\left(\ln n_{a}\right)^{\prime}+\left[m_{a} v^{2} /\left(2 T_{a}\right)-3 / 2\right]\left(\ln T_{a}\right)^{\prime}$ and $F_{2 a}=\left(\ln n_{a} u\right)^{\prime}+\left[m_{a} v^{2} /\left(2 T_{a}\right)-5 / 2\right]\left(\ln T_{a}\right)^{\prime}$, where we denote $\psi$-derivatives by ' . Using $\mathbf{b} \cdot\left(\mathbf{E}_{1}+c^{-1} \mathbf{v} \times \mathbf{B}_{1}\right) \approx E_{\|}-c^{-1} \mathbf{v}_{\perp} \cdot \nabla A_{\|}$we find

$$
\begin{aligned}
& \left(\mathbf{E}_{1}+\frac{1}{c} \mathbf{v} \times \mathbf{B}_{1}\right) \cdot \nabla_{v} f_{s}=-\frac{m_{e} u v_{\|} f_{M e}}{T_{e}} F_{2 e} \\
& \times\left\{\frac{I}{\Omega_{e}}\left(E_{\|}-\frac{\mathbf{v}_{\perp}}{c} \cdot \nabla A_{\|}\right)+\frac{m_{e}}{e_{e}}\left[c \frac{\partial \phi_{1}}{\partial \zeta}+\frac{d A_{\zeta}}{d t}\right.\right. \\
& \left.\left.-\mathbf{v} \cdot\left(\nabla \psi \frac{\partial A_{\psi}}{\partial \zeta}+\nabla \theta \frac{\partial A_{\theta}}{\partial \zeta}+\nabla \zeta \frac{\partial A_{\zeta}}{\partial \zeta}\right)\right]\right\}-\frac{m_{e} u f_{M e}}{T_{e}}\left(E_{\|}-\frac{\mathbf{v}_{\perp}}{c} \cdot \nabla A_{\|}\right) \\
& -\left(\frac{m_{e}}{T_{e}}\right)^{2} v_{\|} u f_{M e} \mathbf{v} \cdot\left[\nabla \phi_{1}+\frac{1}{c}\left(\nabla \psi \frac{\partial A_{\psi}}{\partial t}+\nabla \theta \frac{\partial A_{\theta}}{\partial t}+\nabla \zeta \frac{\partial A_{\zeta}}{\partial t}\right)\right]
\end{aligned}
$$

Defining

$$
g_{e}=f_{1 e}+\frac{e_{e} \phi_{1}}{T_{e}} f_{M e}\left(1-\frac{m_{e} u v_{\|}}{T_{e}}\right)+A_{\zeta} f_{M e}\left[F_{1 e}-\frac{m_{e} u v_{\|}}{T_{e}} F_{2 e}\right]
$$

and, combining (3), (4) and (6), we derive the kinetic equation governing this portion of the distribution function

$$
\begin{aligned}
& \frac{d g_{e}}{d t}=-\frac{e_{e}}{m_{e}}\left(1-\frac{m_{e} u v_{\|}}{T_{e}}\right)\left[\frac{\partial \phi_{1}}{\partial t}-\frac{\mathbf{v}}{c} \cdot\left(\nabla \psi \frac{\partial A_{\psi}}{\partial t}+\nabla \theta \frac{\partial A_{\theta}}{\partial t}+\nabla \zeta \frac{\partial A_{\zeta}}{\partial t}\right)\right] \frac{\partial f_{M e}}{\partial E} \\
& -c\left[\frac{\partial \phi_{1}}{\partial \zeta}-\frac{\mathbf{v}}{c} \cdot\left(\nabla \psi \frac{\partial A_{\psi}}{\partial \zeta}+\nabla \theta \frac{\partial A_{\theta}}{\partial \zeta}+\nabla \zeta \frac{\partial A_{\zeta}}{\partial \zeta}\right)\right] \\
& \times\left[F_{1 e}-\frac{m_{e} u v_{\|}}{T_{e}} F_{2 e}\right]+\left(E_{\|}-\frac{\mathbf{v}_{\perp}}{c} \cdot \nabla A_{\|}\right)\left[R c \frac{m_{e} u v_{\|}}{T_{e}} f_{M e} F_{2 e}+\frac{e_{e}}{T_{e}} u f_{M e}\right]
\end{aligned}
$$

To obtain this equation we made use of the fact that $d_{t}$ vanishes when acting on $\partial_{E} f_{* e}$ and $\partial_{\psi *} f_{* e}$, and thus it approximately vanishes when acting on $\partial_{E} f_{M e}$ and $\partial_{\psi} f_{M e}$, as finite orbit width effects are neglected. Furthermore, we used $\mathbf{v} \cdot \nabla \phi_{1}=\left(d_{t}-\partial_{t}\right) \phi_{1}$.

Following a procedure similar to that in [5] we can derive the gyro-kinetic equation. After a transformation to gyro center variables, a gyro-phase average of the kinetic equation (8) is performed and finite orbit width effects are neglected where appropriate. We neglect compressional magnetic perturbations as small in the normalized pressure $\mathbf{b} \cdot \mathbf{B}_{1} / B_{0} \sim \beta_{i} e \phi_{1} / T_{i}$. For electrons we also neglect FLR corrections. We note that $\mathbf{v}_{\perp} \cdot \nabla A_{\|}$vanishes upon gyro-phase averaging and the $\propto E_{\|} R c$ term in the last line of (8) is small in the gyrokinetic ordering and therefore can be neglected. We arrive at the result

$$
\begin{aligned}
& \frac{\partial g_{e}}{\partial t}+\left(v_{\|} \mathbf{b}+\mathbf{v}_{d e}\right) \cdot \nabla g_{e}=\frac{e_{e}}{T_{e}}\left(\frac{\partial \phi_{1}}{\partial t}-\frac{v_{\|}}{c} \frac{\partial A_{\|}}{\partial t}\right)\left(1-\frac{m_{e}}{T_{e}} u v_{\|}\right) f_{M e} \\
& -c f_{M e}\left(\frac{\partial \phi_{1}}{\partial \zeta}-\frac{v_{\|}}{c} \frac{\partial A_{\|}}{\partial \zeta}\right)\left[F_{1 e}-\frac{m_{e} u v_{\|}}{T_{e}} F_{2 e}\right]+\frac{e_{e}}{T_{e}} f_{M e} u E_{\|},
\end{aligned}
$$

where the electron drift velocity of the guiding center in the equilibrium magnetic field is $\mathbf{v}_{d e}$, and the parallel component of the fluctuating vector potential is $A_{\|}=$ $I\left(A_{\zeta}+A_{\theta} / q\right) /\left(B R^{2}\right)$. This form of $A_{\|}$follows from assuming straight field line 
coordinates, i.e. $\mathbf{B}_{0} \cdot \nabla \theta=I / q R^{2}$ with $q$ a flux function. The full fluctuating electron distribution $f_{1 e}$ and $g_{e}$ are related by

$$
g_{e}=f_{1 e}+\frac{e_{e} \phi_{1}}{T_{e}} f_{M e}\left(1-\frac{m_{e}}{T_{e}} u v_{\|}\right) .
$$

where a term $A_{\zeta} f_{M e}\left[F_{1 e}-m_{e} u v_{\|} F_{2 e} / T_{e}\right]$ has been neglected as small in our ordering. The magnitude of this term will be quantified in the beginning of Sec. 3 when a specific form for the perturbations will be assumed.

We keep FLR corrections when deriving the ion gyrokinetic equation to obtain the usual result

$$
\begin{aligned}
& \frac{\partial g_{i}}{\partial t}+\left(v_{\|} \mathbf{b}+\mathbf{v}_{d i}\right) \cdot \nabla g_{i} \\
& =\frac{e_{i}}{T_{i}} f_{M i}\left(\frac{\partial\left\langle\phi_{1}\right\rangle}{\partial t}-\frac{v_{\|}}{c} \frac{\partial\left\langle A_{\|}\right\rangle}{\partial t}\right)-c f_{M i}\left(\frac{\partial\left\langle\phi_{1}\right\rangle}{\partial \zeta}-\frac{v_{\|}}{c} \frac{\partial\left\langle A_{\|}\right\rangle}{\partial \zeta}\right) F_{1 i}
\end{aligned}
$$

where $\langle\cdot\rangle$ denotes a gyro-phase average at fixed guiding center position, and the relation between $g_{i}$ and $f_{1 i}$ is given by

$$
g_{i}=f_{1 i}+\frac{e_{i} \phi_{1}}{T_{i}} f_{M i}
$$

where again, a term $A_{\zeta} f_{M i} F_{1 i}$ has been neglected for our ordering.

So far we have derived the linearized electromagnetic gyrokinetic equations, where we allow for a parallel flow of electrons. We assumed large aspect ratio and small beta, and neglected $\mathbf{E}_{0}$ and electron FLR effects, but otherwise the equations (9, 12) are still rather general. In the next section we shall derive a dispersion relation for the high mode number kink modes, where further approximations regarding the magnetic geometry and the mode structure will be made.

\section{Dispersion relation of the high mode number kink mode}

In this section we assume a flute like mode structure for the perturbed quantities $\propto \exp (-i \omega t+i m \theta-i n \zeta)$, where the fluctuations are elongated along magnetic field lines with $m \approx n q \gg 1$, where $q \sim 1$ is the safety factor. From $\nabla \cdot \mathbf{A}_{1}=0$ we find $A_{\theta} \approx A_{\zeta} r^{2} n /\left(R^{2} m\right)$ and thus $A_{\|} \approx I A_{\zeta}\left[1+r^{2} /(q R)^{2}\right] /\left(B R^{2}\right) \approx A_{\zeta} / R$ and b $\cdot \nabla=i I(m-n q) /\left(q B R^{2}\right) \approx i k_{\|}$with $k_{\|}$the parallel wave number. The size of $A_{\|} \sim k_{\|} c \phi_{1} / \omega$ is set by assuming $E_{\|} \approx 0$. We consider a pure plasma, but allow the ion charge number $Z$ to be different from 1 . By assuming $L$ to be comparable with the minor radius of the device, we can neglect magnetic drifts as small in $\epsilon$ as compared to the diamagnetic drifts when deriving our dispersion relation. The justification of neglecting magnetic drifts, which is a good approximation at long wavelengths, will be further discussed towards the end of this section. Finally, the mode frequency $\omega$ is assumed to be comparable or larger than the diamagnetic frequency $\sim\left(n_{i} c T_{i} / e_{i}\right)\left(\ln p_{i}\right)^{\prime}$. We find that the $A_{\zeta}$ terms neglected in the derivation of (10) and (12) are smaller than the $\phi_{1}$ terms by $\left(k_{\|} v_{i} / \omega\right)\left(\rho_{i} / L\right)(q / \epsilon)$, with $\rho_{i}=v_{i} / \Omega_{i}$ the ion gyro radius. 
From quasineutrality we have

$$
0=\sum_{a} e_{a} \int d^{3} v\left[g_{a}+\left(f_{1 a}-g_{a}\right)\right]
$$

where $f_{1 a}-g_{a}$ for electrons and ions are given in (10) and (12). The velocity integrals of the $f_{1 a}-g_{a}$ parts of the distributions are straightforward to evaluate. Using quasineutrality for the unperturbed densities, equation (13) reduces to

$$
\sum_{a} e_{a} \int d^{3} v g_{a}=\frac{e_{e}^{2} n_{e}}{T_{e}}\left(1+\frac{Z T_{e}}{T_{i}}\right) \phi_{1},
$$

where $Z=-e_{i} / e_{e}$ denotes the ion charge number. The fluctuating parallel current $j_{\|}$ is given by

$$
j_{\|}=\sum_{a} e_{a} \int d^{3} v v_{\|}\left[g_{a}+\left(f_{1 a}-g_{a}\right)\right]
$$

Again, the velocity integrals of $f_{1 a}-g_{a}$ can be readily evaluated using the relations (10) and (12), to find

$$
\sum_{a} e_{a} \int d^{3} v v_{\|} g_{a}=j_{\|}-e_{e} n_{e} u \frac{e_{e}}{T_{e}} \phi_{1}
$$

Note that the velocity integrals of (13,16) are taken at fixed particle position, while the $g_{a}$ appearing in the gyrokinetic equations are functions of the guiding center position.

When the magnetic drifts are neglected the gyrokinetic equations are of the form

$$
\partial_{t} g_{a}+v_{\|} \mathbf{b} \cdot \nabla g_{a}=\mathrm{RHS}_{a}
$$

where RHS $a$ represents the right hand sides of (9) and (11) for $a=e$ and $i$, respectively. We integrate (17) over the velocity space at fixed particle position and sum over species to find

$$
\frac{\partial}{\partial t}\left(\sum_{a} e_{a} \int d^{3} v g_{a}\right)+\mathbf{B} \cdot \nabla\left(\sum_{a} \frac{e_{a}}{B} \int d^{3} v v_{\|} g_{a}\right)=\sum_{a} e_{a} \int d^{3} v \mathrm{RHS}_{a} .
$$

The velocity integrals are to be performed in $E$ and $\mu$ variables, and the Jacobian is $B /\left|v_{\|}\right|$, leading to the form of the second term of (18). For electrons the integral of $\mathrm{RHS}_{e}$ gives

$$
\begin{aligned}
& e_{e} \int d^{3} v \mathrm{RHS}_{e}=\frac{e_{e}^{2} n_{e}}{T_{e}}\left(\frac{\partial \phi_{1}}{\partial t}+\frac{u}{c} \frac{\partial A_{\|}}{\partial t}\right) \\
& -e_{e} n_{e} c \frac{\partial \phi_{1}}{\partial \zeta} \frac{\partial \ln n_{e}}{\partial \psi}-e_{e} n_{e} u \frac{\partial A_{\|}}{\partial \zeta} \frac{\partial \ln \left(n_{e} u\right)}{\partial \psi}+\frac{e_{e}^{2}}{T_{e}} n_{e} u E_{\|}
\end{aligned}
$$

For ions we need to account for FLR effects. We use $\int d^{3} v f_{M i}\left\langle\phi_{1}\right\rangle J_{0}\left(k_{\perp} v_{\perp} / \Omega_{i}\right)=$ $\phi_{1} \int d^{3} v f_{M i} J_{0}^{2}\left(k_{\perp} v_{\perp} / \Omega_{i}\right) \approx n_{i} \phi_{1}\left[1-\left(k_{\perp} \rho_{i}\right)^{2} / 2\right]$ to evaluate the integral for the ions through first order in $\alpha_{i}=\left(k_{\perp} \rho_{i}\right)^{2} / 2$, where $k_{\perp}$ is the perpendicular wave number. As a result we find

$$
e_{i} \int d^{3} v \operatorname{RHS}_{i}=\left(1-\alpha_{i}\right) \frac{e_{i}^{2} n_{i}}{T_{i}} \frac{\partial \phi_{1}}{\partial t}-c e_{i} n_{i}\left[\left(1-\alpha_{i}\right) \frac{\partial \ln p_{i}}{\partial \psi}-\frac{\partial \ln T_{i}}{\partial \psi}\right] \frac{\partial \phi_{1}}{\partial \zeta}
$$


We then substitute (14), (16), (19) and (20) into (18) and use quasineutrality to find

$$
\begin{aligned}
& \mathbf{B} \cdot \nabla\left(\frac{j_{\|}}{B}-\frac{e_{e} n_{e} u}{B} \frac{e_{e}}{T_{e}} \phi_{1}\right)=-\alpha_{i} \frac{e_{e}^{2} n_{e}}{T_{e}} \frac{Z T_{e}}{T_{i}} \frac{\partial \phi_{1}}{\partial t} \\
& +\frac{e_{e}^{2} n_{e}}{T_{e}} \frac{u}{c} \frac{\partial A_{\|}}{\partial t}+\alpha_{i} e_{i} n_{i} c \frac{\partial \ln p_{i}}{\partial \psi} \frac{\partial \phi_{1}}{\partial \zeta}-e_{e} n_{e} u \frac{\partial A_{\|}}{\partial \zeta} \frac{\partial \ln \left(n_{e} u\right)}{\partial \psi}+\frac{e_{e}^{2} n_{e}}{T_{e}} u E_{\|} .
\end{aligned}
$$

Realizing that the $\mathbf{b} \cdot \nabla \phi_{1}$ term on the left hand side of (21) together with the $\partial_{t} A_{\|}$ term on the right hand side of (21) exactly cancel with the $E_{\|}$term, we can simplify to obtain

$$
i k_{\|} j_{\|}=i \omega \tau \alpha_{i} \frac{e_{e}^{2} n_{e}}{T_{e}} \phi_{1}-i n c e_{i} n_{i} \alpha_{i} \phi_{1} \frac{\partial \ln p_{i}}{\partial \psi}+i n u e_{e} n_{e} A_{\|} \frac{\partial \ln \left(n_{e} u\right)}{\partial \psi}
$$

where we employ the mode structure $\exp (-i \omega t+i m \theta-i n \zeta)$ and define $\tau=$ $Z T_{e} / T_{i}$. Introducing the background current gradient and the pressure gradient driven diamagnetic frequencies

$$
\omega_{* e}^{j}=\frac{n c T_{e}}{e_{e}} \frac{\partial \ln j_{0}}{\partial \psi}, \quad \omega_{* i}^{p}=\frac{n c T_{i}}{e_{i}} \frac{\partial \ln p_{i}}{\partial \psi},
$$

with $j_{0}=e n_{e} u$ and $p_{i}=n_{i} T_{i}$, and multiplying (22) by $-i k_{\|} c T_{e} /\left(e_{e}^{2} n_{e}\right)$ we find

$$
\frac{k_{\|}^{2} c T_{e}}{e_{e}^{2} n_{e}} j_{\|}=\alpha_{i} \tau k_{\|} c \phi_{1}\left(\omega-\omega_{* i}^{p}\right)+\omega_{* e}^{j} k_{\|} u A_{\|} .
$$

Then we employ the parallel Ampère's law $k_{\perp}^{2} A_{\|}=(4 \pi / c) j_{\|}$, and recall $\beta_{i}=8 \pi p_{i} / B^{2}=$ $\left(v_{i} / v_{A}\right)^{2}$, where $v_{A}=\left[B^{2} /\left(4 \pi m_{i} n_{i}\right)\right]^{1 / 2}$ is the Alfvén speed, to rewrite the left hand side of (24) as $\tau\left(v_{i} k_{\|}\right)^{2} \alpha_{i} A_{\|} / \beta_{i}$. We focus on the $E_{\|} \approx 0$ limit, that is $\phi_{1} \approx \omega A_{\|} /\left(k_{\|} c\right)$. This approximation will be justified at the end of this section. We use this relation to eliminate $\phi_{1}$ from (24) in favor of $A_{\|}$, and then divide by $\tau\left(k_{\perp} \rho_{i}\right)^{2} / 2$ to obtain the dispersion relation

$$
\omega\left(\omega-\omega_{* i}^{p}\right)=\frac{\left(v_{i} k_{\|}\right)^{2}}{\beta_{i}}-\frac{\omega_{* e}^{j} k_{\|} u}{\tau \alpha_{i}} .
$$

The solution of (25) for the mode frequency is then

$$
\omega=\frac{\omega_{* i}^{p}}{2} \pm\left[\left(\frac{\omega_{* i}^{p}}{2}\right)^{2}+\frac{\left(v_{i} k_{\|}\right)^{2}}{\beta_{i}}-\frac{2 \omega_{* e}^{j} k_{\|} u}{\tau\left(k_{\perp} \rho_{i}\right)^{2}}\right]^{1 / 2} .
$$

This result is consistent with Equation (15) of [18], which was derived in a shearless slab geometry. In the $\omega_{* i}^{p} \ll \omega$ limit (26) reduces to

$$
\omega= \pm\left(\frac{\left(v_{i} k_{\|}\right)^{2}}{\beta_{i}}-\frac{2 \omega_{* e}^{j} k_{\|} u}{\tau\left(k_{\perp} \rho_{i}\right)^{2}}\right)^{1 / 2}
$$

For a given wave number if the electron flow speed $u$ or the normalized pressure $\beta_{i}$ is sufficiently small the first term dominates on the right hand side of (27), and the solution is an Alfvén wave with purely real frequency $\omega^{2} \approx\left(v_{A} k_{\|}\right)^{2}$. However, for high enough $\beta_{i}, u$ and $\omega_{* e}^{j}$ the second term might exceed the first and, depending on the relative sign of $k_{\|}$and $\mathrm{u}$, (27) describes either a pair of stable modes with purely real frequencies or a purely growing and a purely damped mode. 
For the rest of this section we will be concerned with the purely growing mode driven by the current gradient. Clearly, decreasing the perpendicular wave number of the mode increases the growth rate of the mode. Since the first term in (27) is quadratic and the second term is linear in $k_{\|}$, there is an optimal value of the parallel wave number, $k_{\| o}$, where the mode has the highest growth rate, $\gamma$. When the plasma parameters and the perpendicular wave number are fixed the optimum is

$$
k_{\| o}=\frac{u \beta_{i} \omega_{* e}^{j}}{v_{i}^{2}\left(k_{\perp} \rho_{i}\right)^{2} \tau}
$$

and the growth rate corresponding to $k_{\|_{o}}$ is

$$
\gamma_{o}=\frac{u \sqrt{\beta_{i}} \omega_{* e}^{j}}{v_{i}\left(k_{\perp} \rho_{i}\right)^{2} \tau} .
$$

When $\omega_{* e}^{j} \sim \omega_{* i}^{p}$ and $\tau \sim 1$, the assumption $\omega_{* i}^{p} \ll|\omega|$ used to obtain (27) is satisfied if $1 \ll u \sqrt{\beta_{i}} /\left(v_{i} \alpha_{i}\right)$. As long as there is a finite plasma beta and electron current, one can always find sufficiently small perpendicular wave number for which this relation is satisfied in the $\rho_{i} / L \rightarrow 0$ limit. In this case, neglecting magnetic drifts in the gyrokinetic equation is also justified as long as the pressure length scale is much smaller than the major radius.

It is shown at the end of Appendix A, the perturbed quasineutrality equation can be written in the form $0=\left[\phi_{1}-\omega A_{\|} /\left(k_{\|} c\right)\right] G_{1}+G_{2}$, where $G_{1}$ is a dimensionless function of order unity (as long as $\omega /\left(k_{\|} v_{e}\right)$ is not too large) and $G_{2}$ is small in $\alpha_{i}$. Thus, neglecting the small correction from $G_{2}$, the approximate quasineutrality equation $0=\left[\phi_{1}-\omega A_{\|} /\left(k_{\|} c\right)\right] G_{1}$ is satisfied either if $G_{1}=0$, or $\phi_{1}-\omega A_{\|} /\left(k_{\|} c\right)=0$, that is if $E_{\|}=0$, which we assumed in deriving (27). The case $G_{1}=0$ includes drift wave solutions and the strongly damped modes corresponding to electrostatic roots of the uniform plasma dispersion relation in the presence of electron flow.

\section{Magnetic shear effects}

To obtain simple analytical results in Section 3 we neglected magnetic drifts and assumed a flute like mode structure (with no radial variation). The mode tends to be more unstable at low perpendicular wave numbers, thus it is appropriate to neglect the magnetic drifts, $\mathbf{v}_{d i} \cdot \mathbf{k}_{\perp} \ll \omega$.

Due to the preceding assumptions, the result (26) is formally the same as what one would obtain solving the problem in a shearless slab geometry [18. The only difference between a torus and a slab is that $\omega_{* e} \propto n$ and $k_{\perp} \rho_{i} \propto n$ have lower limits set by the lowest finite toroidal wave number $n=1$. We note that in a shearless slab there is no such periodicity constraint, and $k_{\perp} \rho_{i}$ can get arbitrarily small (thus $\gamma$ arbitrarily large) for sufficiently large perpendicular wave lengths. This unphysical behavior is partly resolved by taking finite magnetic shear into account, which is needed for the magnetic geometry to be consistent with a substantial parallel current. In this section we will study the consequences of a magnetic shear in slab geometry. 
We choose a coordinate system $\{\hat{x}, \hat{y}, \hat{z}\}$ such that plasma parameters vary in the $\hat{x}$ direction, and consider a mode which is sinusoidally varying in the $\hat{y}$ direction with a corresponding wave number $k_{y}$, while the magnetic field has the form $\mathbf{B}=B\left(\hat{z}+\hat{y} x / L_{s}\right)$. The magnetic shear produces an $x$ variation in $k_{\|}$, namely $k_{\|}(x)=k_{\|}(0)+k_{y} x / L_{s}$, and we choose the origin so that $k_{\|}(0)=0$. We assume that the radial variations of the perturbed quantities are faster than those of the unperturbed ones and $\beta_{i} \ll 1$, thus the $\hat{y}$ component of the electron flow can be neglected $(\mathbf{u}=u \hat{z})$ together with any change in the magnitude of $\hat{z} \cdot \mathbf{B}$.

To obtain a dispersion relation in a sheared geometry we start with (24) and insert parallel Ampére's law together with $\alpha_{i} \rightarrow \rho_{i}^{2}\left(k_{y}^{2}-\partial_{x x}^{2}\right) / 2$ to find

$$
\tau \frac{v_{i}^{2} k_{\|}^{2}}{\beta_{i}} \frac{\rho_{i}^{2}}{2}\left(k_{y}^{2}-\partial_{x x}^{2}\right) A_{\|}=\tau k_{\|} c\left(\omega-\omega_{* i}^{p}\right) \frac{\rho_{i}^{2}}{2}\left(k_{y}^{2}-\partial_{x x}^{2}\right) \phi_{1}+\omega_{* e}^{j} k_{\|} u A_{\|} .
$$

Then, we assume $E_{\|} \approx 0$ to replace $\phi_{1}$ in (30) by $\omega A_{\|} /\left(k_{\|} c\right)$, which is consistent with neglecting $\mathcal{O}\left(\alpha_{i}\right)$ terms in the quasineutrality equation. Taking the $y$-derivative of (30) leads to the dispersion relation in terms of the $x$-component of the perturbed magnetic field, $B_{x}$

$$
-\omega\left(\omega-\omega_{* i}^{p}\right) \frac{\rho_{i}^{2}}{2}\left(k_{y}^{2}-\partial_{x x}^{2}\right)\left(\frac{\hat{B}}{k_{\|}}\right)+\frac{\left(k_{\|} v_{i}\right)^{2}}{\beta_{i}} \frac{1}{k_{\|}} \frac{\rho_{i}^{2}}{2}\left(k_{y}^{2}-\partial_{x x}^{2}\right) \hat{B}-\frac{k_{\|} u \omega_{* e}^{j}}{\tau} \frac{\hat{B}}{k_{\|}}=0,
$$

where $\hat{B}$ is defined by $B_{x}=\hat{B}(x) \exp \left(-i \omega t+i k_{y} y\right)$. The dispersion relation is essentially the same as in shearless geometry, except for the linear $x$-dependence of $k_{\|}$, and that the replacement $\partial_{x} \rightarrow i k_{x}$ cannot be made.

Recalling $k_{\|}=k_{y} x / L_{s}$ and introducing the dimensionless "radial" coordinate $X=k_{y} x$, (31) can be rewritten in the form

$$
X\left(\partial_{X X}^{2}-1\right) \hat{B}-\lambda\left(\partial_{X X}^{2}-1\right)(\hat{B} / X)-\sigma \hat{B}=0
$$

where $\lambda=\omega\left(\omega-\omega_{* i}^{p}\right) L_{s}^{2} \beta_{i} / v_{i}^{2} \approx \omega^{2} L_{s}^{2} \beta_{i} / v_{i}^{2}$ and $\sigma=-2 L_{s} \beta_{i} u \omega_{* e}^{j} /\left(\tau k_{y}^{2} \rho_{i}^{2} v_{i}^{2}\right)$. The boundary conditions for this eigenvalue problem in $\lambda$ are given by the requirement that $\hat{B}(|X| \rightarrow \infty) \rightarrow 0$. In (32) $\sigma$ represents the drive and $\lambda<-\beta_{i}\left[\omega_{* i}^{p} L_{s} /\left(2 v_{i}\right)\right]^{2}$ corresponds to an instability $\operatorname{Im}(\omega)>0$. During the analysis of the radial eigenmodes we shall neglect $\left(\omega_{* i}^{p} / \omega\right)^{2} \ll 1$ corrections, and refer to the $\lambda<0$ solutions as unstable and the $\lambda=0$ solutions as marginally stable modes. We will retain $\omega_{* i}^{p}$ corrections in Section 5, We note that reversing the sign of $\sigma$, that is, the relative sign of $u$ and $k_{\|}$, leads to the same eigenvalues, and the corresponding eigenfunctions satisfy $\left.\hat{B}(X)\right|_{-\sigma}=\left.\hat{B}(-X)\right|_{\sigma}$. Thus, henceforth we will analyze solutions corresponding to $\sigma>0$, without loss of generality.

For small values of $X,(32)$ is dominated by the $\lambda$ term, that is solved by $\hat{B}=c_{1} X \exp (X)+c_{2} X \exp (-X)$. Accordingly, the solutions are either linear or quadratic in $X$ around $X=0$. For high values of $X$ the first term dominates (32), leading to the an exponential asymptotic behavior $\hat{B}(X) \propto \exp ( \pm X)$, consistent with the boundary conditions. To solve numerically we rewrite the eigenvalue problem (32) for $F=\hat{B}(X) / X$ as

$$
X\left(X F^{\prime \prime}+2 F^{\prime}-X F\right)-\lambda\left(F^{\prime \prime}-F\right)-\sigma X F=0
$$


and discretize it using a second order finite difference scheme. We set $(X F)^{\prime}-X F=0$ as the negative $X$, and $(X F)^{\prime}+X F=0$ for the positive $X$, boundary conditions to select solutions with the appropriate asymptotic behavior. Then we numerically search for the eigenvalues $\lambda$ and eigenfunctions $F$ of the system for a given $\sigma$.
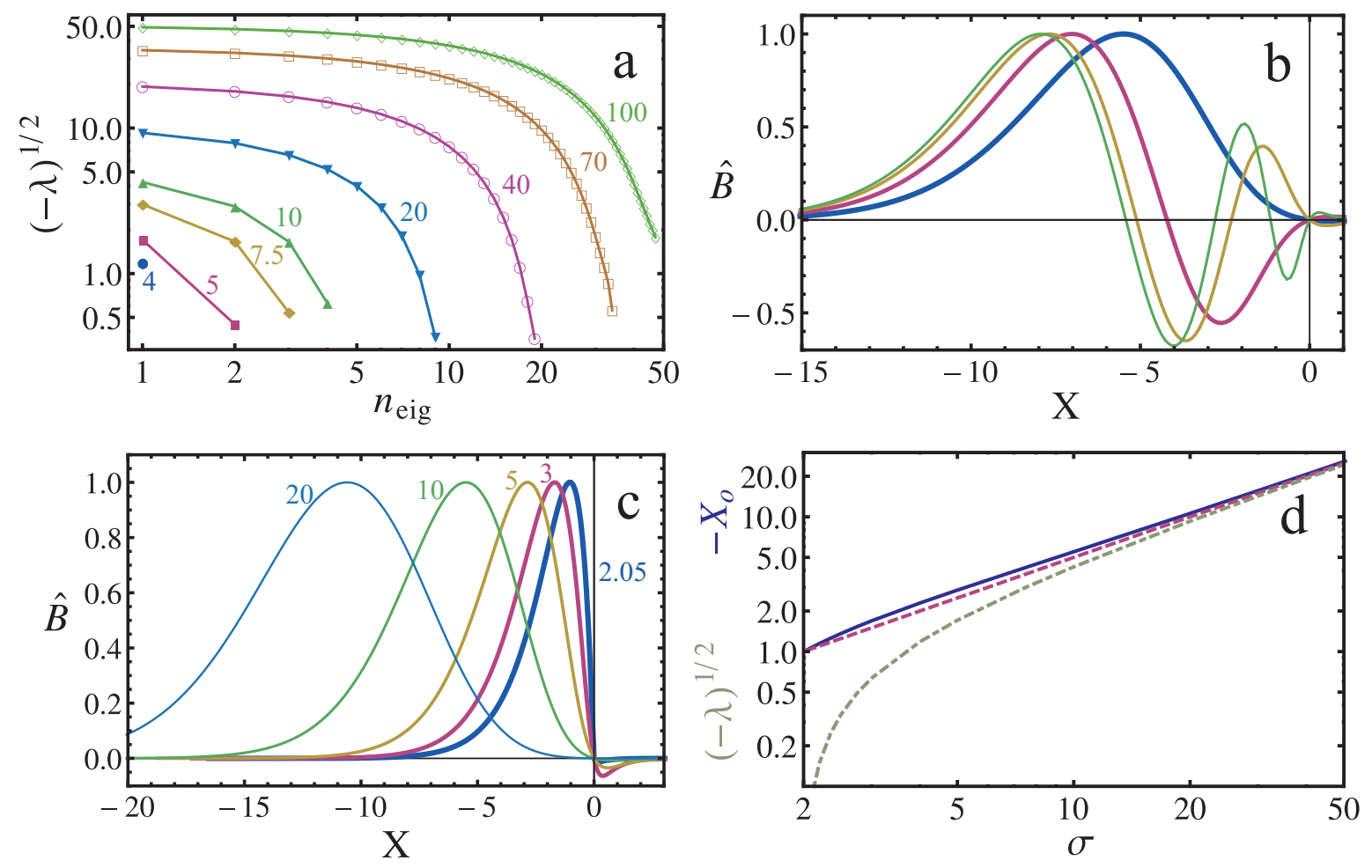

Figure 1. Solutions of the eigenvalue problem (32). (a) Normalized growth rates $(-\lambda)^{1 / 2}$ of unstable modes corresponding to $\sigma=\{4,5,7.5,10,20,40,70,100\}$. The number of unstable modes $\max \left(n_{\text {eig }}\right)$ increases with $\sigma$. (b) Radial eigenmodes $B(X)$ of the four unstable mode at $\sigma=10$. Lower growth rates correspond to more oscillatory structure. (c) The most unstable radial eigenmodes for $\sigma=\{2.05,3,5,10,20\}$. The distance of the location of the maximum of $|\hat{B}(X)|$, from $X=0$ increases with $\sigma$. (d) Solid curve: $-X_{o}$, where $X_{o}$ is the location of the maximum of $|\hat{B}(X)|$ for the most unstable mode at a given value of $\sigma$. Dash-dotted curve: normalized growth rate $(-\lambda)^{1 / 2}$ of the most unstable mode. Dashed curve: $\sigma / 2$.

Figure 1 shows solutions of the eigenvalue problem (32). We find that as the drive $\sigma$ is increased, more and more unstable eigenfunctions appear, as illustrated in Figure 1 a showing the normalized growth rates $(-\lambda)^{1 / 2}$ of all the unstable eigenmodes for different values of $\sigma$. On the x-axis of Figure $19, n_{\text {eig }}$ denotes the ordinal number of the unstable modes, with $n_{\text {eig }}=1$ corresponding to the most unstable mode for each value of $\sigma$. In fact, a new unstable mode appears as $\sigma$ exceeds $2 N$ for every positive integer $N$. In particular, no unstable mode exists for $|\sigma| \leq 2$. Note that in Figure 1 the marginally stable $(\lambda=0)$ modes for even values of $\sigma$ are not shown.

For $\sigma=2 N$, the marginally stable $(\lambda=0)$ solutions of (32) are of the form $\hat{B}(X)=X \exp (-X)_{1} F_{1}(1+\sigma / 2,2 ; 2 X)=X \exp (X) P_{\sigma}(X)$ for $X \leq 0$ and $\hat{B}(X)=0$ 
for $X>0$. Here, ${ }_{1} F_{1}$ denotes the Kummer confluent hypergeometric function, and $P_{\sigma}$ is a polynomial with only positive coefficients $\left(P_{2}=1, P_{4}=1+X, P_{6}=1+2 X+2 X^{2} / 3\right.$, ...). The derivative of the marginally stable solutions is discontinuous at $X=0$, however it is resolved by a boundary layer at +0 for $\sigma=2 N+\delta$ with an arbitrarily small $\delta>0$ and a corresponding small eigenvalue $\lambda$. The boundary layer connects the $X \leq 0$ solution vanishing at $X=0$, to a solution $\propto \exp (-X)[1+2 X \exp (2 X) \operatorname{Ei}(-2 X)]$ for $X>0$, which is finite at $X=0$. Here, $\operatorname{Ei}(x)=\mathcal{P} \int_{-\infty}^{x} \exp (t) / t d t$ (for real values of $x$ ) denotes the Exponential integral, where $\mathcal{P}$ indicates that the principal value is to be used for $x \geq 0$. No marginally stable solution to (32) exists if $|\sigma| \neq 2 N$, since in this case $X \exp (-X)_{1} F_{1}(1+\sigma / 2,2,2 X)$ becomes divergent at $X \rightarrow-\infty$, and the $\hat{B}(X \rightarrow-\infty)=0$ boundary condition cannot be met.

When more than a single unstable eigenmode exists $(\sigma>4)$, the ones with lower growth rates exhibit a more oscillatory radial structure, as illustrated in $1 \mathrm{~b}$ showing the four unstable modes for $\sigma=10$. In particular, the most unstable mode (corresponding to the thickest curve in $1 \mathrm{~b}$ ) does not change sign in the region $X<0$, while all the other unstable modes do. This is consistent with the behavior of the marginally stable modes, since for increasing $N$ the number of roots of $P_{\sigma}(X)$ increases.

The amplitude $|\hat{B}|$ of the most unstable eigenmode has a maximum close to the radial location where $k_{\|}(X)$ would maximize the local dispersion relation (27), that is $k_{\|}(X) \approx k_{\| o}$ with the optimal wave number $k_{\| o}$ given in (28).

In terms of $X$, the location of $k_{\|}(X)=k_{\| o}$ scales as $X_{o}=-\sigma / 2$ according to the local theory. As shown in $1 \mathrm{~d}$, the location of the maximum amplitude (solid curve, representing $-X_{o}$ ) follows this expectation (dashed curve, $\sigma / 2$ ) quite well. In the strongly driven $(\sigma \gg 1)$ limit the normalized growth rate $(-\lambda)^{1 / 2}$ of the most stable eigenmode (dash-dotted curve in 11d) approaches the optimal value, $\gamma_{o}$ given by (29). This value corresponds to $(-\lambda)^{1 / 2} \rightarrow \sigma / 2$. However, $\sigma=2$ gives $(-\lambda)^{1 / 2}=0$ (that is, no unstable mode) in the sheared slab model, while the local theory would predict a finite growth rate equivalent with $(-\lambda)^{1 / 2}=1$.

In conclusion, considering magnetic shear sets a stability limit in terms of the drive at $\sigma=2$ in contrast to the shearless model that predicts instability when $\beta_{i}$, the current gradient and the flow speed $u$ are finite. In the shearless case the mode is always allowed to pick the optimal parallel wave number.

The stability criterion of the mode $|\sigma|<2$ is equivalent to that of the high mode number kink modes. Using the relations $u=j_{0} /\left(e n_{e}\right), L_{s}=q R / s, s=(r / q) d q / d r$, $k_{y}=n q / r$ and $n=m / q$, with $m$ the poloidal mode number, together with the definitions of $\sigma$ and $\omega_{* e}^{j}$, one can rewrite the stability criterion $|\sigma|<2$ as

$$
\frac{4 \pi r}{c B_{\theta}}\left|\frac{d j_{0}}{d r}\right|<2 m\left|\frac{q^{\prime}}{q}\right|,
$$

as obtained from the magnetohydrodynamic energy principle in [1] - see Equation (2.29) therein. 


\section{Mode characteristics in toroidal geometry}

In this section the high mode number kink mode investigated in Sections 3 and 4 is studied numerically using the gyrokinetic code GS2. GS2 is free from the simplifying assumptions made in Section 4, except for the radial locality and the scale separation $k_{\|} \ll k_{\perp}$. In the low-flow version of GS2 extra terms related to neoclassical corrections to the non-fluctuating part of the distribution function and the electrostatic potential are implemented for momentum transport studies, as discussed in [17]. These quantities are specified as inputs, normally calculated by the neoclassical code NEO [19]. This infrastructure can in principle be used to include any modification to the non-fluctuating part of the distribution over a velocity range of a few thermal speeds. We use it to include $f_{s}$, as defined after (2), or more sophisticated Spitzer functions, to study the effect of the induced electric field on instabilities. Normally, we include only a parallel flow in GS2 simulations, instead of a full Spitzer function since the results are insensitive to the detailed form.

First we consider the parametric dependences of the mode frequency and the growth rate, and compare GS2 simulations to predictions of the sheared slab model (SSM) (31). The SSM results are obtained by choosing the most unstable eigenmode from the numerical solution of (33). We use a 200 point radial grid, the extent of which is adapted to the expected width of the eigenfunctions depending on the value of $\sigma$.

The scans are performed about the following set of base-line parameters: $u / v_{i}=1$, $\beta_{i}=0.01, a / L_{u}=3, a / L_{T i}=a / L_{T e}=a / L_{n}=0, k_{y} \rho_{i}=0.15, a / R=0.1, r / a=0.5$, $s=1$, and $q=10$, where $d \ln u / d r=-1 / L_{u}, d \ln n_{e} / d r=-1 / L_{n}, d \ln T_{e} / d r=-1 / L_{T e}$, and $d \ln T_{i} / d r=-1 / L_{T i}$. We set the density and temperature gradients to zero to avoid the appearance of the usual gradient driven modes (otherwise, for this $\beta_{i}$, magnetic shear and $L_{n} \sim a \sim L_{T}$ kinetic ballooning modes appear and pollute the results, as in [20]). Then the only instability drive is due to the gradient of the flow speed. The radial gradient of the flow speed in the Ohmic current is due to density and electron temperature gradients, thus our settings are not physically consistent. However, by artificially choosing the parameters we obtain a cleaner comparison between theory and simulations.

The binormal wave number and the aspect ratio are chosen to be small so that magnetic drifts are not expected to affect the results significantly. For a typical Gs2 simulation only an extended poloidal angle range of $\theta=(-\pi, \pi)$ is kept and 80 grid points along the field line are used, since the eigenfunctions of strongly driven modes are highly oscillatory and very localized in $\theta$. The simulations use 20 untrapped pitch angle- and 14 energy grid points. We neglect collisions and compressional magnetic perturbations.

Figure 2 shows various parameter scalings around the baseline parameter set. In a strongly driven situation $(|\sigma| \gg 2)$, the growth rate is expected to be close to (29), which helps in interpreting the numerical results. Since $\omega_{* e}^{j} \propto k_{y}$, and $k_{\perp} \sim k_{y}$, we expect a $1 / k_{y}$ dependence of the growth rate, which is observed in Fig. 2 a. Magnetic 

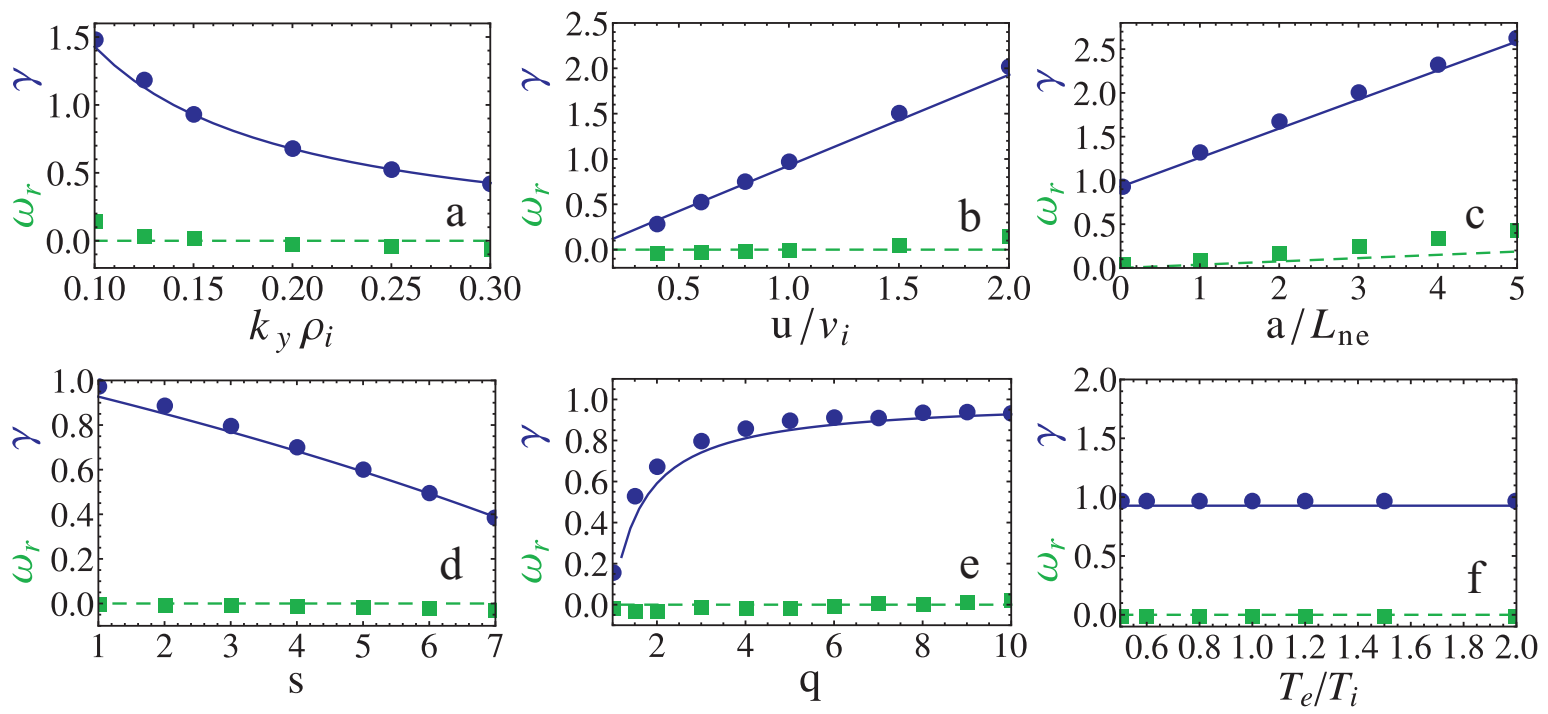

Figure 2. Parametric scalings of the growth rate $\gamma$ (solid line and circle markers) and real frequency $\omega_{r}$ (dashed lines and square markers) of the high mode number kink mode (given in $v_{i} / a$ units). Markers represent GS2 simulations and lines are results of the sheared slab model. The figures depict the dependence on the following: (a) binormal wave number $k_{y} \rho_{i}$, (b) electron flow velocity $u / v_{i}$, (c) density gradient $a / L_{n}$, (d) magnetic shear $s$, (e) safety factor $q$, and (f) temperature ratio $T_{e} / T_{i}$.

drifts should be more important towards higher wave numbers. The good agreement remains between GS2 and the SSM even at $k_{y} \rho_{i}=0.3$ due to the very large aspect ratio $R / r=20$. The growth rate is expected to increase linearly with the flow speed and the mode should be stable at $u=0$ and this behavior is seen in Fig. 2b. Similarly, the growth rate should exhibit the linear dependence on $a / L_{n}$ as shown, where the mode is unstable at $a / L_{n}=0$ due to the finite gradient in the flow speed, see Fig. 2r.

To translate magnetic geometry parameters from the toroidal geometry of GS2 to a sheared slab we use $1 / L_{s}=s / q R$. Although the local model can be used to explain certain parametric dependences of the mode, it cannot provide predictions for the $L_{s}$ dependence. However we know that as $\sigma \propto L_{s}$ drops below 2 due to a decreasing $L_{s}$, the mode should be completely stabilized. Thus we expect increasing $s$ should reduce the growth rates, as seen in Fig. 2 d. Clearly, $q$ should have the opposite effect as $s$, since $L_{s} \propto q / s$. Indeed, Fig. 2 2 shows that the mode is stabilized with decreasing $q$. Also, when the mode is strongly driven, $|\sigma| \gg 2$, the growth rate should become independent of $L_{s}$, since the mode approaches the local result. Hence, there is a saturation in the $q$-dependence of $\gamma$ towards higher values of $q$. When $\beta_{i}$ and $k_{y} \rho_{i}$ are held fixed the growth rate given in (29) normalized to $v_{i} / a$ is independent of $T_{e} / T_{i}$. The insensitivity of the result to the temperature ratio is demonstrated in Fig. $2 \mathrm{~d}$.

The real part of the frequency $\omega_{r}$ is proportional to the ion diamagnetic frequency $\omega_{* i}^{p}$, which should be zero in almost all the scalings of Fig. 2, since the ion pressure gradient is zero. The only exception is the density gradient scaling, Fig. $2 \mathbb{k}$; where $\omega_{r}$ 
should increase linearly with $a / L_{n}$. Although, we find the right trend $\omega_{r} \propto a / L_{n}$, GS2 produces higher values than the slab model. The reason for this discrepancy is likely that the mode is not purely kink anymore, but instead develops some kinetic ballooning character due to the finite pressure gradient drive.

There are small deviations from the $\omega_{r}=0$ result of the slab model in the GS2 simulations in Fig. 2a, b and d-f. These may be the result of the magnetic drift effects neglected in the slab model, but also, they may also represent the finite accuracy of the simulations. In certain cases, when $\sigma$ is very high, making the parallel mode structure very oscillatory, exceptionally high parallel resolutions were necessary in Gs2 to achieve the accuracy presented in Fig. 2 (for example 140 grid points in $\theta$ ).

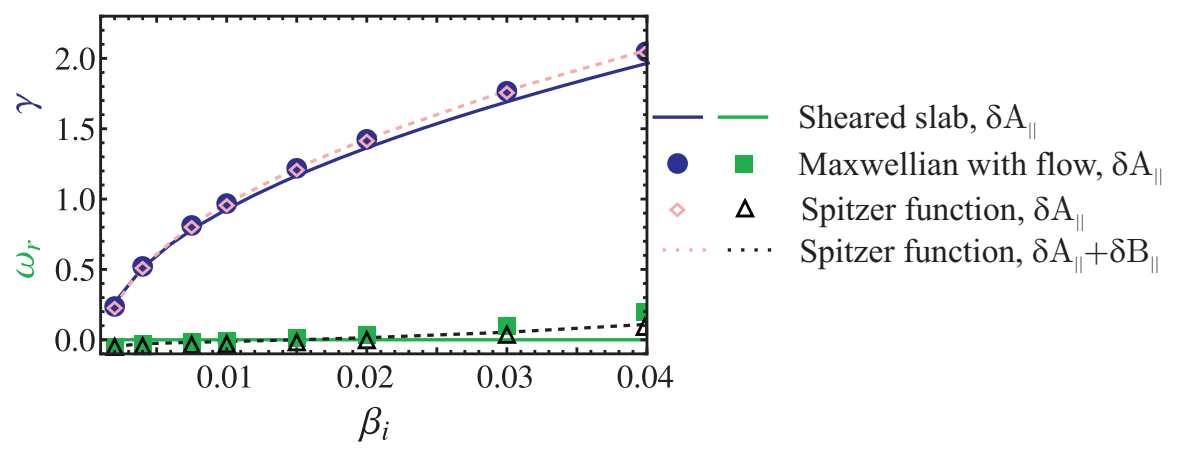

Figure 3. $\beta_{i}$ scaling of the growth rate $\gamma$ (upper curves and points) and real frequency $\omega_{r}$ of the high mode number kink mode (given in $v_{i} / a$ units). The solid lines are sheared slab model results, the dotted curves and symbols are Gs2 simulation results computed using a shifted Maxwellian electron distribution (full symbols), using a Spitzer function keeping only $A_{\|}$fluctuations (empty symbols), and using a Spitzer function keeping both $A_{\|}$and $B_{\|}$fluctuations (dotted curves).

The expected $\sqrt{\beta_{i}}$ dependence of the growth rate of the high mode number kink modes is reproduced, as seen in Fig. 3. Apart from the sheared slab results (solid) lines, Fig. 3 shows GS2 simulations of different levels of sophistication. In the simplest case the non-fluctuating electron distribution is modeled as a Maxwellian with a finite parallel flow velocity (shown with solid symbols). It is interesting to see that when the shifted Maxwellian is replaced by a Spitzer function with the same flow speed but considerably more complicated velocity space structure (given by (B4) and (B8) of [21]), the results (empty symbols) remain practically unchanged, especially for the growth rates. Spot checks for different plasma parameters show the same behavior. This demonstrates that the velocity structure of the non-fluctuating part of the electron distribution is unimportant, and that only its parallel flow speed matters for the kink mode. All the simulations presented herein include only $A_{\|}$perturbations except those shown with the dotted lines in Fig. 3. We find that in the strongly driven cases corresponding to our baseline set of parameters, compressional magnetic perturbations have no significant impact on the mode frequencies.

We note that the normalized ideal magnetohydrodynamic drive, often referred to 
as the MHD inertial-layer width [8] is qualitatively different for the high-m kink modes studied here and for the $m=1$ mode [22]. This drive, which determines the ideal MHD growth rate of the mode, is $\epsilon^{2}$ small in the $m=1$ case (as compared to $m \neq 1$ ) making the near marginally stable mode sensitive to non-ideal effects such as collisional or collisionless reconnection. Although the simulations shown in Figs. 2 and 3 are collisionless and they do not resolve scales of the electron skin depth, these high-m modes are so strongly unstable due to the ideal MHD drive that they are not expected to be sensitive to physics happening in small layers around the $k_{\|}=0$ surface.
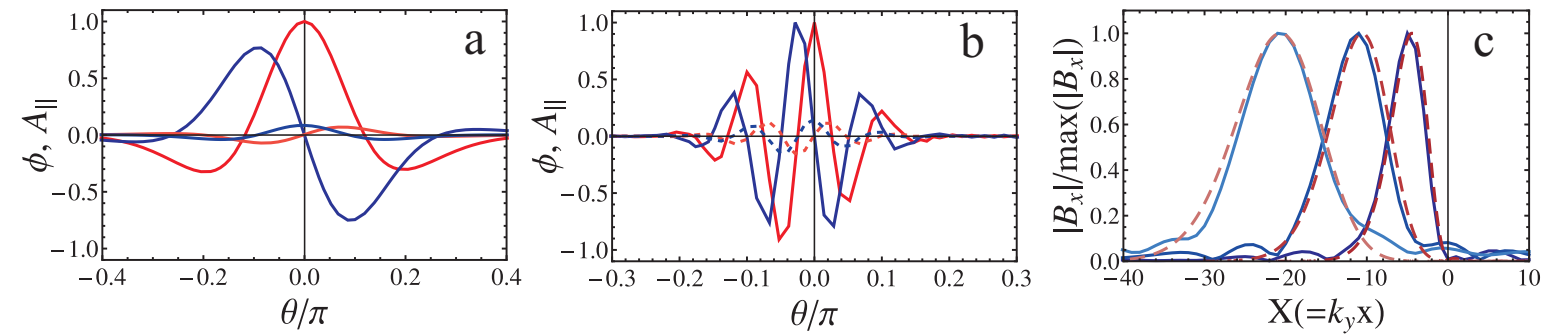

Figure 4. a-b: Parallel mode structures from GS2 simulations. Solid curves are $\phi$, and dashed curves are $A_{\|}$; red and blue curves correspond to the real and imaginary parts, respectively; and (a) $\beta_{i}=0.004$, (b) $\beta_{i}=0.02$. (c) The radial mode structures in the SSM (dashed curves) and calculated from GS2 parallel mode structures (solid curves); $\beta_{i}=\{0.004,0.01,0.02\}$, the corresponding curves peak at increasing $|X|$ values.

Typical parallel mode structures are shown in Figures $4 \mathrm{a}$ and b. These simulations are done for the baseline parameters with varying plasma beta; $\beta_{i}=0.004$ and 0.02 in $4 \mathrm{a}$ and b, respectively. Note that the kink drive, $\left|j_{0}\right|^{\prime} \propto\left|n_{e} u\right|^{\prime}$, is still finite due to the finite density gradient. Increasing $\beta_{i}$, corresponds to more oscillatory parallel structures (larger $k_{\|}$), as expected from (28), and an increasing amplitude of the magnetic component of the fluctuations. In the sheared slab geometry, the parallel wave number increases away from the resonant surface (recall $k_{\|}=k_{y} x / L_{s}$ ).

The Fourier transform of the sheared slab problem in the $x$ coordinate can lead to an equation that is equivalent to the problem in ballooning representation with a coordinate along the magnetic field line [23]. More precisely, the radial eigenfunction in the sheared slab, $\hat{B}(X)$, is related to the ballooning eigenfunction, $B_{B}(\theta)$, by $\hat{B}(X) \propto \int_{-\infty}^{\infty} d \theta e^{i \theta X} B_{B}(-\theta / s)$. Figure 4le shows that the kink modes considered here have this same property. It compares the variation of the radial mode structure [the magnitude of $\hat{B}(X)$ ] in sheared slab calculations (dashed lines), with the transform of the ballooning mode variation obtained from Gs2 (solid), for different values of $\beta_{i}$. The solid line peaking the closest to (and furthest away from) the rational surface correspond to the ballooning eigenfunction in Fig. $4 a$ (and b, respectively). The "ballooning character" of the eigenfunctions, that is, their localization around $\theta=0$, is simply a consequence of how a mode with a finite radial extent appears in ballooning representation, rather than a result of a poloidal dependence in the drive of the mode. In particular it is not a magnetic drift effect. As the radial extent of $\hat{B}(X)$ increases with increasing $\sigma \propto \beta_{i}$, 
the equivalent $B_{B}(\theta)$ becomes more and more localized around $\theta=0$ according to the properties of the Fourier transformation.

We note that from the sheared slab dispersion relation (30) and $A_{\|}=k_{\|} c \phi_{1} / \omega$ the long wavelength ballooning equations solved by GS2 can be recovered using the replacements $i k_{\|} \rightarrow(q R)^{-1} \partial_{\theta}$ and $i k_{y} \hat{y}+\hat{x} \partial_{x} \rightarrow i k_{y} \hat{y}+i k_{y} s \theta \hat{x}$.

\section{Discussion and conclusions}

We have developed a procedure for modeling current gradient driven kink instabilities in a tokamak with GS2 gyrokinetic simulations and compared the results to the analytical expressions we derived.

We find that at sufficiently high current gradient high mode number kink modes are destabilized. The properties of strongly driven kink modes can be understood from simple analytical expressions derived in a shearless magnetic geometry by assuming that the mode chooses an optimal, finite parallel wave number that maximizes its growth rate. In terms of kinetic quantities, the mode is destabilized by high $\beta_{i}$, strong parallel electron flow $u$, high values of $\partial_{\psi}\left(\ln n_{e} u\right)$, and small perpendicular wave numbers.

Since the mode is more unstable for smaller values of the perpendicular wave numbers $k_{\perp}$, magnetic drift effects $\left(\propto \mathbf{k}_{\perp} \cdot \mathbf{v}_{d a}\right)$ are unimportant for describing the stability of the mode. A perhaps more important effect of toroidicity is that there is a lower limit on $k_{\perp}$ set by the lowest finite toroidal mode number $n=1$. However, both the analytical calculations and GS2 assume a scale separation $k_{\|} \ll k_{\perp}$ and disregard global profile and magnetic geometry variations, thus are unable to properly treat low mode number magnetohydrodynamic modes. Therefore the stability limit, which we derive based on kinetic theory, coincides with the magnetohydrodynamic stability limit for high mode number kink modes [1]. In the sheared slab magnetic geometry we find that the mode is strongly asymmetric, being localized on one side with respect to a resonant $\left(k_{\|}=0\right)$ surface. The parallel wave number corresponding to the radial location of the highest amplitude is close to the one that maximizes the growth rate in the local theory. The number of unstable radial eigenmodes increases with increasing drive.

We find good agreement between GS2 simulations and analytical estimates both in terms of the parametric dependences of the growth rates and mode frequencies, and in terms of eigenmode structure. The large aspect ratio and small $k_{y} \rho_{i}$ limit of high mode number kink modes may be used as a simple test case for linear validation of electromagnetic gyrokinetic codes when current drive is to be modeled. By comparing kink modes assuming a Maxwellian electron distribution with a parallel flow and alternatively a Spitzer function departure from a Maxwellian as a drive we demonstrate that the exact velocity structure of the non-fluctuating electron distribution function is unimportant for the mode. Only the parallel flow speed of electrons matters.

For modes that are electrostatic in nature, an electron flow - even when comparable

to the ion thermal speed - is not expected to significantly modify their stability. The circulating electrons which can flow along the field lines are close to be adiabatic, 
and their already small non-adiabatic response is only modified by an even smaller correction from the flow. Without showing specific GS2 results, we remark that we have found practically no effect on ion- and electron temperature gradient modes for typical plasma parameters even when the plasma $\beta$ and the electron flow speed exceeds their experimentally relevant range in the simulations.

In a screw-pinch geometry it is known that, if an ideal magnetohydrodynamic mode is unstable at a given finite poloidal mode number $m_{0}$, it should be even more unstable at all mode numbers $m$ satisfying $1 \leq m<m_{0}$ [24]. Therefore, the trend of increasing growth rate with decreasing $k_{\perp}$ is not terminated until the lowest wave number allowed in the system. Consequently, if the plasma is globally stable to low mode number kink modes, it should be stable for all mode numbers. However, since a similar theorem has not been proven in toroidal geometry, the relevance of high mode number kink modes in tokamaks is unclear, and should be the subject of future investigations. Toward this end, the research herein demonstrates that suitably modified gyrokinetic codes can be used to investigate current driven or kink instabilities in tokamaks. A local code such as GS2 permits the modeling of only high wave number modes, but it has the important advantage that it can effectively model the nonlinear evolution of these modes, which is a topic for future studies.

\section{Acknowledgments}

The authors are thankful to Jesus Ramos, Jack Connor, Jeff Freidberg, and Jim Hastie for several fruitful discussions on MHD related problems, and to Choongki Sung for providing experimental parameters. This work was funded by the European Communities under Association Contract between EURATOM and Vetenskapsrådet (VR), and by the US Department of Energy grant at DE-FG02-91ER-54109 at MIT. The first author is grateful for the financial support of VR.

\section{Appendix A. Quasineutrality}

To derive the explicit form of the quasineutrality equation from (14) we write it as

$$
0=e_{a} \int d^{3} v g_{e}-\frac{e_{e}^{2} n_{e}}{T_{e}} \phi_{1}+e_{i} \int d^{3} v g_{i}-\frac{e_{i}^{2} n_{i}}{T_{i}} \phi_{1},
$$

where the integrals are taken at fixed particle position. First we will evaluate the electron contribution to quasineutrality, i.e. the first two terms of (A.1). We neglect the magnetic drifts in (91), replace time derivatives by $-i \omega$, toroidal derivatives by $-i n$, write $E_{\|}=-i k_{\|} \phi_{1}+i \omega A_{\|} / c$, and then divide the equation by $-i \omega+i k_{\|} v_{\|}$, to obtain

$$
\begin{aligned}
& g_{e}=\frac{e_{e}}{T_{e}} f_{M e}\left(1-\frac{m_{e}}{T_{e}} u v_{\|}\right) \frac{\omega\left(\phi_{1}-\frac{v_{\|}}{c} A_{\|}\right)}{\omega-k_{\|} v_{\|}} \\
& -n c f_{M e} \frac{\phi_{1}-\frac{v_{\|}}{c} A_{\|}}{\omega-k_{\|} v_{\|}}\left[F_{1 e}-\frac{m_{e}}{T_{e}} u v_{\|} F_{2 e}\right]+f_{M e} \frac{e_{e}}{T_{e}} u k_{\|} \frac{\phi_{1}-\frac{\omega A_{\|}}{k_{\|} c}}{\omega-k_{\|} v_{\|}} .
\end{aligned}
$$


The integral $e_{e} \int d^{3} v g_{e}$ in (A.1) can be directly evaluated in terms of the plasma dispersion function, using that

$$
Z(\xi)=\int_{-\infty}^{\infty} \frac{d x}{\sqrt{\pi}} \frac{\exp \left(-x^{2}\right)}{x-\xi}
$$

where the integration is done along the Landau contour. After a straightforward calculation we find that the electron contribution to the dispersion relation is

$$
\begin{aligned}
& -\frac{T_{e}}{e_{e}^{2} n_{e}} \int d^{3} v f_{1 e} \\
& =\left(\phi_{1}+\bar{A}\right)\left[\left(1+\xi_{e} Z\left(\xi_{e}\right)\right)\left(1-\frac{\omega_{* e}}{\omega}\right)-\frac{\omega_{* e} \eta_{e}}{\omega}\left(\xi_{e}^{2}+Z\left(\xi_{e}\right)\left(\xi_{e}^{3}-\xi_{e} / 2\right)\right)\right] \\
& +\left(\phi_{1}+\bar{A}\right) \frac{U k_{\|}}{\left|k_{\|}\right|}\left\{Z\left(\xi_{e}\right)-2 \xi_{e}\left[\left(1+\xi_{e} Z\left(\xi_{e}\right)\right)\left(1-\frac{\omega_{* e}}{\omega}\left(1+\eta_{u}-\eta_{e}\right)\right)\right.\right. \\
& \left.\left.-\frac{\omega_{* e} \eta_{e}}{\omega}\left(\xi_{e}^{2}+Z\left(\xi_{e}\right)\left(\xi_{e}^{3}-\xi_{e} / 2\right)\right)\right]\right\}+\phi_{1} \frac{\omega_{* e}}{\omega},
\end{aligned}
$$

where we introduced $\xi_{a}=\omega /\left(\left|k_{\|}\right| v_{a}\right)$, the normalized flow speed $U=u / v_{e}$, the diamagnetic frequency $\omega_{* a}=\left(n c T_{a} / e_{a}\right) \partial_{\psi} n_{a}$, and $\bar{A}=-\omega A_{\|} /\left(k_{\|} c\right)$.

Once the ion magnetic drifts are neglected, the gyro-averages $\langle\cdot\rangle$ are replaced by $J_{0}\left(k_{\perp} v_{\perp} / \Omega_{i}\right)$, and the $\zeta$-derivatives are written in terms of $\omega_{* i}, g_{i}$ from (11) can be easily expressed as the familiar form

$$
g_{i}=f_{M i} \frac{e_{i}}{T_{i}}\left(\phi_{1}-\frac{v_{\|}}{c} A_{\|}\right) J_{0}\left(\frac{k_{\perp} v_{\perp}}{\Omega_{i}}\right) \frac{\omega-\omega_{* i}\left[1+\eta_{i}\left(\frac{m_{i} v_{i}^{2}}{2 T_{i}}-\frac{3}{2}\right)\right]}{\omega-k_{\|} v_{\|}} .
$$

When we evaluate the velocity integral for ions in (A.1) we expand in the FLR parameter, writing $J_{0}^{2}\left(k_{\perp} v_{\perp} / \Omega_{i}\right)=J_{0}^{2}\left(k_{\perp} \rho_{i} v_{\perp} / v_{i}\right) \approx 1-\alpha_{i}\left(v_{\perp} / v_{i}\right)^{2}$, where we recall the definition $\alpha_{i}=\left(k_{\perp} \rho_{i}\right)^{2} / 2$. The ion contribution to the dispersion relation, normalized to $-e_{i}^{2} n_{i} / T_{i}$, is obtained to be

$$
\begin{aligned}
& -\frac{T_{i}}{e_{i}^{2} n_{i}} \int d^{3} v f_{1 i} \\
& =\left(1-\alpha_{i}\right)\left(\phi_{1}+\bar{A}\right)\left[\left(1+\xi_{i} Z\left(\xi_{i}\right)\right)\left(1-\frac{\omega_{* i}}{\omega}\right)-\frac{\omega_{* i} \eta_{i}}{\omega}\left(\xi_{i}^{2}+Z\left(\xi_{i}\right)\left(\xi_{i}^{3}-\xi_{i} / 2\right)\right)\right] \\
& +\alpha_{i} \frac{\omega_{* i} \eta_{i}}{\omega}\left[\left(\phi_{1}+\bar{A}\right) \xi_{i} Z\left(\xi_{i}\right)+\bar{A}\right]+\alpha_{i} \phi_{1}\left(1-\frac{\omega_{* i}}{\omega}\right)+\phi_{1} \frac{\omega_{* i}}{\omega} .
\end{aligned}
$$

Note that (A.4) and (A.6) contain the contributions from the adiabatic responses. When the perturbed quasineutrality equation (A.1) is formed the contributions from $\phi_{1} \omega_{* e} / \omega$ and $\phi_{1} \omega_{* i} / \omega$ [the last terms in (A.4) and (A.6) , respectively] cancel for a pure plasma, due to quasineutrality $e_{e} n_{e}+e_{i} n_{i}=0$ and $\left(\ln n_{e}\right)^{\prime}=\left(\ln n_{i}\right)^{\prime}$.

Due to the high electron thermal speed $\xi_{e}$ is typically small. As long as $\xi_{e}$ is not much larger than unity there are $\mathcal{O}(1)$ terms multiplying $\phi_{1}+\bar{A}$ in the electron contribution to quasineutrality (A.4). In the ion contribution (A.6), the terms in the last line, which cannot be factorized by $\phi_{1}+\bar{A}$ are multiplied by $\alpha_{i}$ that is assumed to be small in our expressions. In conclusion, the quasineutrality equation has an order unity part that can be factorized by $\phi_{1}-\omega A_{\|} /\left(k_{\|} c\right)$, and the rest is small in $\alpha_{i} \phi_{1}$. This 
means that to satisfy quasineutrality, either $\phi_{1}$ and $\omega A_{\|} /\left(k_{\|} c\right)$ should nearly cancel or the coefficient factorized by $\phi_{1}-\omega A_{\|} /\left(k_{\|} c\right)$ should be close to zero.

\section{References}

[1] Kadomtsev B B and Pogutse O P 1970 Rev. Plasma Phys. Vol5 249, ed. Leontovich M A.

[2] Kadomtsev B B 1975 Sov. J. Plasma Phys. 1389.

[3] Basu B and Coppi C 1981 Phys. Fluids 24465.

[4] Drake J F 1978 Phys. Fluids 211777.

[5] Catto P J 1978 Plasma Phys. 20719.

[6] Frieman E A and Chen L 1982 Phys. Fluids 25502.

[7] Naitou H, Tsuda K, Lee W W and Sydora R D 1995 Phys. Plasmas 2, 4257.

[8] Mishchenko A and Zocco A 2012 Phys. Plasmas 19, 122104.

[9] Mishchenko A, Könies A and Hatzky R 2009 Phys. Plasmas 16082105.

[10] Deng W, Lin Z and Holod I 2012 Nucl. Fusion 52023005.

[11] Lin Z, Hahm T S, Lee W W, Tang W M and White R B 1998 Science 281 №5384, 1835.

[12] http://gene.rzg.mpg.de

[13] Pueschel M J, Jenko F, Told D and Büchner J 2011 Phys. Plasmas 18112102.

[14] Numata R, Dorland W, Howes G G, Loureiro N F, Rogers B N and Tatsuno T 2011 Phys. Plasmas 18112106.

[15] Numata R, Howes G G, Tatsuno T, Barnes M, Dorland W 2010 J. Comp. Phys. 2299347.

[16] Kotschenreuther M, Rewoldt G and Tang W M 1995 Comp. Phys. Comm. 88128.

[17] Barnes M, Parra F I, Lee J P, Belli E A, Nave M F F and White A E 2013 Phys. Rev. Lett. 111 055005 .

[18] Sperling J L and Bhadra D K 1979 Plasma Phys. 21225.

[19] Belli E A and Candy J 2008 Plasma Phys. Control. Fusion 50095010.

[20] Pusztai I, Catto P J, Parra F I and Barnes M 2013 Proceedings of the 40th EPS Conference on Plasma Physics P4.156.

[21] Pusztai I and Catto P J 2010 Plasma Phys. Control. Fusion 52 075016; the coefficient of $Z$ in $a_{0}$ in (B8) should read $1208 \sqrt{2}$.

[22] Rosenbluth M N, Dagazian R Y and Rutherford P H 1973 Phys. Fluids 161894.

[23] Connor J W, Hastie R J and Taylor J B 1979 Proc. R. Soc. Lond. A 3651.

[24] Newcomb W A 1960 Annals of Physics 10232. 